\title{
A Motion Compensation Strategy for Airborne Repeat-Pass SAR Data
}

\author{
Virginia Brancato, Marc Jäger, Rolf Scheiber, and Irena Hajnsek, Fellow, IEEE
}

\begin{abstract}
Generating accurate repeat-pass interferometric airborne SAR products demands the precise compensation of the 3-D motion of the aircraft. This requires to estimate and correct small residual motion errors within the accuracy limits of the sensor navigation sub-system, e.g. using residual motion compensation algorithms. Because of their data-driven nature, the performance of these algorithms severely degrades for heavily decorrelated interferograms.

This letter proposes a generic residual motion error (RME) estimation and compensation strategy optimally estimating RME in a stack of SAR acquisitions, where some interferometric pairs are strongly affected by decorrelation. The algorithm works even if the whole scene decorrelates, as long as the coherence magnitude is reasonably high over short temporal and spatial baselines. The approach entails correcting the navigation data of each slave image of a one-to-many interferometric network with a cumulative correction. The summation is over the results of a precursory application of a general data-driven residual motion compensation algorithm (e.g. multisquint) to interferometric pairs for which the impact of interferometric decorrelation is marginal (i.e. small temporal and/or spatial baselines). Compared to other RME correction strategies, the main appeal of the proposed approach lies in the simplicity of its implementation. The overall methodology is tested on a zero-baseline time series acquired at L-band by the DLR's airborne system F-SAR.
\end{abstract}

Index Terms-Motion compensation (MoCo), residual motion errors estimation, repeat-pass, interferometry, processing, synthetic aperture radar (SAR)

\section{INTRODUCTION}

Spaceborne repeat-pass radar interferometry (InSAR) and differential interferometric SAR (DInSAR) are established techniques applied in the generation of Digital Elevation Models (DEMs) and for measuring deformations of the Earth surface [1]. As a consequence of the stable trajectory of the spacecraft, SAR image focusing does not introduce undesired artefacts due to uncompensated, azimuth time-variant inaccuracies in the assumed sensor position [2]. By contrast, the accuracy of airborne repeat-pass interferometric products is

V. Brancato was with the Institute of Environmental Engineering, Swiss Federal Institute of Technology (ETH) Zurich, 8093 Zurich, Switzerland. Currently, she is with the Jet Propulsion Laboratory, California Institute of Technology, Pasadena, CA 91109 USA and with the the Department of Earth System Science, University of California, Irvine, CA 92697, USA (e-mail: virginia.brancato@gmail.com)

M. Jäger and R. Scheiber are with the Microwaves and Radar Institute of the German Aerospace Center (DLR), 82234 Oberpfaffenhofen, Germany (e-mails: marc.jaeger@dlr.de, rolf.scheiber@dlr.de)

I. Hajnsek is with the Microwaves and Radar Institute of the German Aerospace Center (DLR) and also with the Institute of Environmental Engineering, Swiss Federal Institute of Technology (ETH), 8093 Zurich, Switzerland (e-mail: irena.hajnsek@dlr.de)

Manuscript received ?; revised ?. severely impaired by such inaccuracies, affecting the navigation data input to SAR processing in all three dimensions [2]. SAR imagery processed with imperfect navigation data are said to be affected by residual motion errors, i.e. residual sensor position inaccuracies of 1-5 centimetres [3].

The lack of a sub-centimetre accuracy of the current navigation systems (e.g. INS, GPS) makes residual motion error compensation mandatory for accurate airborne SAR interferometry, as such residual inaccuracies introduce significant phase errors when forming SAR interferograms [3]. These spurious phase patterns usually appear as low-frequency undulations in the azimuth direction [4]. To mitigate these effects, residual motion compensation algorithms (e.g. multisquint) are used [5]. The main drawback of these algorithms is to rely on a reasonably high coherence magnitude in the interferogram under consideration and typically they exhibit suboptimal performances when the interferograms are highly decorrelated. As a figure of merit for the case of DInSAR, the interferometric phase noise needs to be below $3^{\circ}$ to reach a millimetre accuracy at L-band [4]. Low interferometric coherence can be induced by large differences in the acquisition geometry [1] and/or by changes in the scatterers arrangements and/or dielectric properties [1]. This letter presents a refined processing strategy tailored to optimally estimate residual motion errors in stacks of acquisitions, where some interferometric combinations are strongly affected by decorrelation. The proposed approach extends the interferometric SAR processing chain described in [5] by introducing a post-processing residual motion error (RME) estimation algorithm. It consists in the computation of a cumulative motion error as the sum of accurate RME estimates obtained by applying a general data-driven residual motion compensation algorithm to interferometric pairs marginally affected by decorrelation (i.e. those with short temporal and/or spatial baselines). This correction is used to update the navigation data of each slave acquisition of a one-to-many interferometric network and the SAR data stack is newly reprocessed. Compared to other techniques [6], the availability of residual motion error techniques makes the proposed approach effortless to implement.

The letter is organized as follows. Section II introduces the processing chain followed by the proposed RME estimation strategy. The overall approach is evaluated in Section III using a zero-baseline time series acquired by the German Aerospace Center (DLR)'s airborne system F-SAR at L-band. The performance of the proposed approach is then compared with that of using solely a generic residual motion compensation algorithm (e.g. multisquint) for the estimation of the RMEs. 


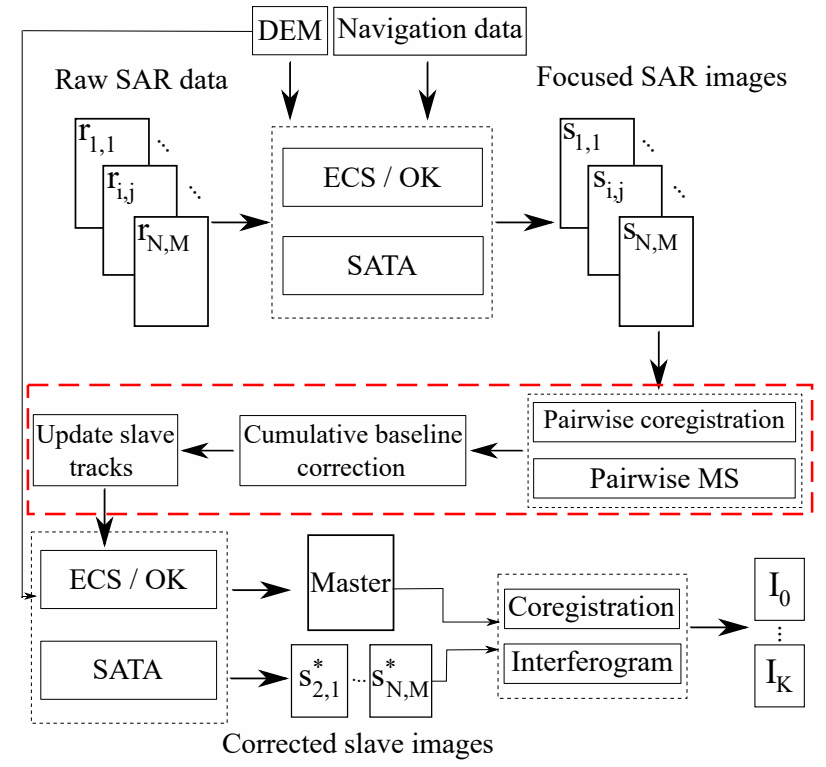

Fig. 1: Block diagram describing the proposed data processing strategy. ECS and OK stands for Extended Chirp Scaling and Omega-K SAR focusing kernels, respectively. SATA abbreviates the SubAperture Topography- and Aperture-dependent motion compensation algorithm. The red box encloses the proposed post-processing RME estimation.

\section{Methods}

\section{A. SAR processing chain}

It is assumed that a set of M multi-baseline SAR images is acquired at each time epoch $t \in(1, \ldots, N)$. This collection of SAR acquisitions can be compactly described through the vector $\mathbf{r}=\left[r_{1,1}, \ldots, r_{1, M}, \ldots, r_{i, j}, \ldots, r_{N, M}\right]$ (see Fig.1). The index $i=1, \ldots, N$ represents the time at which the element $r_{i, j}$ was acquired while $j=1, \ldots, M$ indicates the various flight tracks, considered parallel. Each set of M multi-baseline images is assumed to be acquired roughly simultaneously, in that their maximum temporal baseline can be considered negligible compared to the minimum time separation between the time epochs (e.g. minutes or hours compared to tens of days, months or years).

The relevant parts of the chain used to process the stack of SAR data is shown in Fig.1. Each element of $\boldsymbol{r}$ is focused using a frequency domain kernel (e.g. Extended Chirp Scaling or Omega-K) that incorporates a two-step adaptive MoCo approach using the SubAperture Topography- and Aperturedependent (SATA) algorithm [5]. This approach allows for simultaneously accommodating topographic variations and aircraft movements (along the whole synthetic aperture) taking into account an external DEM [5].

The set of focused SAR images $\mathbf{s}$ can be then interferometrically processed (see Fig.1). The standard interferometric processing includes SAR image coregistration, estimation and correction of the residual baseline errors (RBE), interferogram generation and, optionally, the application of range spectral filtering [5].

The coregistration procedure can be generalized to stacks of

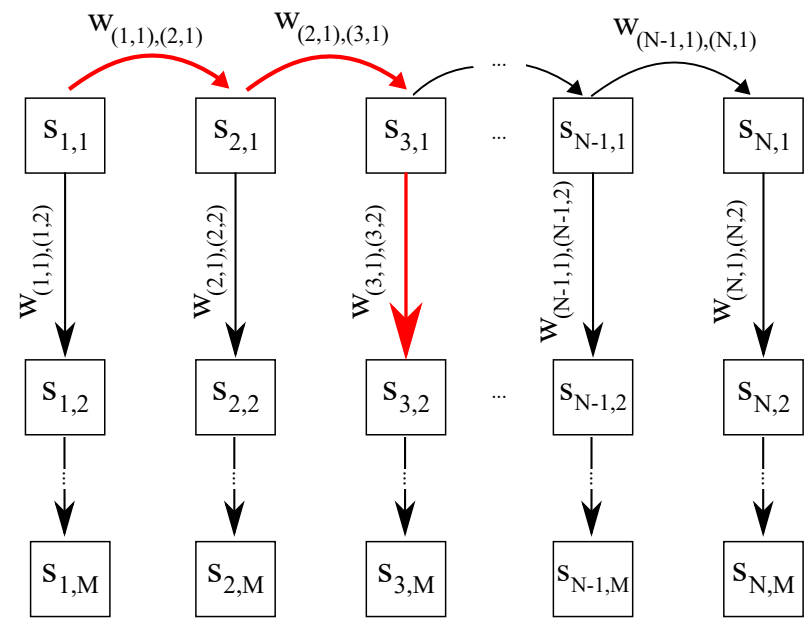

Fig. 2: Oriented, simply connected graph representing the outcome of the pairwise interferometric processing. Each node represents a generic SAR image $s_{i, j}$ while edges are labelled with a matrix $\boldsymbol{w}_{(i, j),(l, k)}$ containing the RBEs estimated with MS for the interferometric pair $\left(s_{i, j}, s_{l, k}\right)$. The red route exemplifies the shortest path connecting the pair $\left(s_{1,1}, s_{3,2}\right)$.

images in different fashions. One solution consists in bringing each element of $s$ into the same geometry as of a single SAR acquisition, forming a one-to-many interferometric network $[1,7]$. Alternatively, one can generate interferograms from all possible, non-trivial coherent combinations of the elements in $s$. However, if reducing the impact of interferometric decorrelation is the prime concern, one may restrict the coregistration to those image pairs where the coherence is likely high, i.e. the pairs in $s$ exhibiting contemporaneously short temporal and spatial baselines. This processing step, concisely indicated in Fig.1 as pairwise coregistration, should guarantee that the resulting interferometric network is fully connected and does not contain isolated clusters, i.e. there should be always a pairwise coregistration/processing edge connecting any given pair of images (see Fig.2). Subsequently, the multisquint (MS) algorithm is applied to the identified pairwise interferometric pairs to obtain an estimate of the underlying RBE in each pairwise interferogram. Since the identified processing pairs are characterised by small temporal and/or spatial baselines, the corresponding RBE estimates result to be particularly accurate [4].

\section{B. Cumulative baseline error correction}

The main outcome of the pairwise processing is an interferometric network, shown, as an example, in Fig.2. To formalize the relations between the various SAR images, the interferometric network is modelled as a directed, simply connected graph $\mathcal{X}=\{\mathcal{V}, \mathcal{E}, \mathcal{W}\}$ consisting of:

- A set of nodes $\mathcal{V}=\left\{s_{1,1}, \ldots, s_{i, j}, \ldots, s_{N, M}\right\}$ representing the focused SAR acquisitions of the data stack.

- A set of edges $\mathcal{E}$ containing ordered pairs of SAR images of the form $\left(s_{i, j}, s_{l, k}\right)$ with $s_{i, j}, s_{l, k} \in \mathcal{V}$. Edges represent the interferometric combinations for which the RBEs have been estimated in the pairwise processing. 
TABLE I: Temporal baselines of the F-SAR data set expressed in days. $\Delta t_{1}$ is measured relative to the image $s_{1,0}$ while $\Delta t_{\text {prec }}$ represents the time difference to the previous image.

\begin{tabular}{ccccc}
\hline Image & Date & Day of the Year & $\Delta t_{1}$ & $\Delta t_{\text {prec }}$ \\
\hline$s_{1,0}$ & 15.05 .2014 & 135 & 0 & 0 \\
$s_{2,0}$ & 22.05 .2014 & 142 & 7 & 7 \\
$s_{3,0}$ & 04.06 .2014 & 155 & 20 & 13 \\
$s_{4,0}$ & 12.06 .2014 & 163 & 28 & 8 \\
$s_{5,0}$ & 18.06 .2014 & 169 & 34 & 6 \\
$s_{6,0}$ & 03.07 .2014 & 184 & 49 & 15 \\
$s_{7,0}$ & 24.07 .2014 & 205 & 70 & 21 \\
\hline
\end{tabular}

- A set of labels $\mathcal{W}$. The generic element $\boldsymbol{w}_{(i, j),(l, k)} \in \mathcal{W}$ is a matrix containing in its columns the RBEs estimates in the horizontal and vertical directions, related to the interferometric pair $\left(s_{i, j}, s_{l, k}\right)$. The subscript $(i, j),(l, k)$ is used hereinafter to concisely indicate the pair of SAR images $\left(s_{i, j}, s_{l, k}\right) \in \mathcal{E}$, i.e. an edge [8].

The graph connectivity, a direct consequence of the pairwise processing, ensures that, for any given pair of nodes, there will be always a set of edges (i.e. a path) connecting them. Due to the graph topology, i.e. a spanning tree, there is only a single path in the graph connecting a given pair of acquisitions [8]. Particularly, the graph may be more concisely described in terms of its connectivity matrix A. A generic element $a_{(i, j),(l, k)}$ of the latter can take only three values:

$$
a_{(i, j),(l, k)}= \begin{cases}1 & \text { if the edge runs from } s_{i, j} \text { to } s_{l, k} \\ -1 & \text { if the edge runs from } s_{l, k} \text { to } s_{i, j} \\ 0 & \text { otherwise }\end{cases}
$$

where $s_{i, j}, s_{l, k} \in \mathcal{V}$. Given that the overall aim of the interferometric processing is a SAR data stack coregistered with respect to a common master acquisition, the accuracy of the interferometric phase can be improved through a post-processing RBE correction strategy, hereafter referred as cumulative baseline error correction. This correction works even in presence of significant decorrelation between some acquisitions of the stack. The strategy entails computing a correction term based on the sum of the RBE estimates obtained from the pairwise application of MS. In particular, the navigation data of a generic slave image $s_{l, k}$ are corrected by the algebraic and component-wise sum of RBEs $\boldsymbol{w}_{(i, j),(l, k)}$ corresponding to the labels of the graph edges forming the shortest path connecting $s_{l, k}$ with the selected master of the data stack $s_{i, j}$ (e.g. red path in Fig.2 where the master and slave acquisitions correspond to $s_{1,1}$ and $s_{3,2}$, respectively). The cumulative correction corresponding to the interferometric pair $\left(s_{i, j}, s_{l, k}\right)$ can be mathematically expressed as:

$$
\boldsymbol{\Delta}_{(i, j),(l, k)}=\sum_{(m, n) \in P} a_{(i, j),(m, n)} \boldsymbol{w}_{(i, j),(m, n)}
$$

where $P \subseteq \mathcal{V}$ indicates the subset of nodes in the graph forming a path from the fixed master acquisition $s_{i, j}$ to the generic slave node $s_{l, k}$. Eq.2 describes the linear programming problem of finding the shortest path in a graph given the source and the destination nodes [8] and it can be solved with wellknown algorithms (e.g. Bellmann-Ford, Dijkstra) [8].

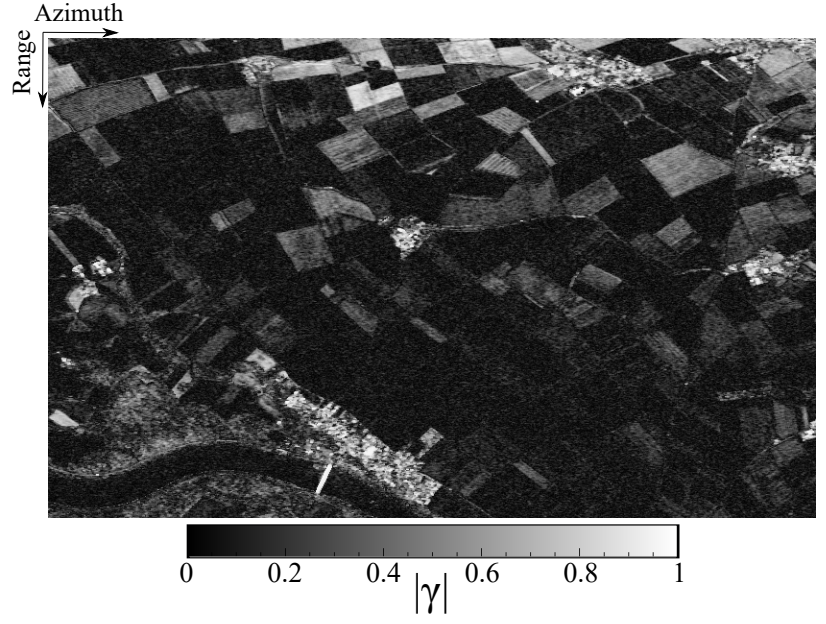

Fig. 3: Coherence magnitude of the interferogram between the images $s_{4,0}$ and $s_{7,0}$. The temporal baseline $\Delta t=42$ days.

After having updated the navigation data of each slave acquisition with the cumulative correction, the entire SAR data stack is reprocessed, starting with the SAR raw data and ending with interferometric coregistration. The interferometric reprocessing is carried out with respect to a single master acquisition but without applying any additional residual baseline error estimation or correction.

For sake of conciseness, the procedure has been implicitly described for the case of a single polarisation channel, but it is straightforward to extend to a set of fully polarimetric SAR data. Assuming that the sensor is well calibrated, the same residual baseline errors can be assumed for all polarisations channels of a single acquisition. Additionally, it should be noticed that any residual motion compensation algorithm can be used for obtaining the estimates of the pairwise RBE, i.e. the proposed approach works with any given data-driven residual motion compensation algorithm. Therefore, the proposed methodology provides an effortless but effective way of generalising the accurate estimation of RBE to SAR data stacks containing some highly decorrelated interferometric pairs (e.g. set of temporally separated tomographic acquisitions).

\section{EXPERIMENTAL RESULTS}

The overall approach (processing strategy and postprocessing residual $\mathrm{MoCo}$ ) has been tested on a zero-baseline data set of seven SAR images, acquired by the DLR's F-SAR system [5] over the agricultural area of Wallerfing, Germany $(48.68 \mathrm{~N}, 12.88 \mathrm{E})$. All the available fully polarimetric acquisitions were recorded at L-band $(1.325 \mathrm{GHz})$ from nearly similar flight tracks at a nominal baseline of $0 \mathrm{~m}$. Table I gives a comprehensive overview of the time intervals between the various sets of images. The test area is rather flat and abundantly dominated by agricultural fields whose growth and vitality may drastically reduce the available degree of interferometric coherence (see Fig.3).

To test the proposed approach, the available data set has been processed in two different fashions:

- direct processing: each interferometric slave has been processed as reported in [5] by solely applying MS for 


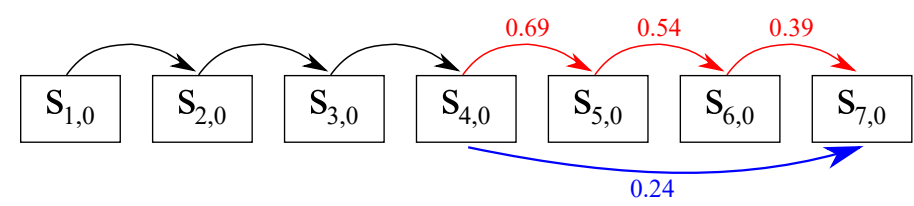

Fig. 4: Pairwise (red) and direct (blue) processing of the FSAR data set. Numbers indicate the mean coherence magnitude for the corresponding interferometric pairs.

the estimation of the RBEs with respect to a common master, selected as the acquisition $s_{4,0}$ (see Fig.4). This will be simply referred as direct approach, hereafter.

- graph processing: the data set has been pairwise processed leading to the interferometric network of Fig.4. Subsequently, the navigation data of each slave image are updated with their corresponding cumulative baseline correction, applied at a SAR raw data level. The data set is reprocessed with respect to a single master, assumed to be the image $s_{4,0}$. This strategy will be referred as graph-based approach in the following.

For sake of conciseness, we focus on the direct and graphbased processing of a single interferometric pair, i.e. $\left(s_{4,0}\right.$, $\left.s_{7,0}\right)$. Their coherent combination is markedly affected by decorrelation, as shown in Fig.3.

Fig.5 shows the RBE estimations projected on the line-of-sight $\left(\boldsymbol{w}_{\text {los }}\right)$, horizontal $\left(\boldsymbol{w}_{y}\right)$, and vertical $\left(\boldsymbol{w}_{z}\right)$ directions obtained for both the direct (Fig. 5 blue line) and the graph-based (Fig. 5 red line) processing strategies. Particularly, the latter corresponds to the sum of the pairwise RBE estimations of the red graph path reported in Fig.4. The result of the summation corresponds to the term correcting the navigation data of the acquisition $s_{7,0}$ i.e., the proposed post-processing residual baseline correction.

Figs.6-a)-b) show the interferometric phase between $\left(s_{4,0}, s_{7,0}\right)$ processed with the direct and graph-based approach, respectively. Additionally, Fig.6-c) shows the phase difference between the slave image processed with both processing strategies and it includes the phase contribution from a linear phase component in azimuth and a constant baseline offset in range, both estimated with a DEM. This long-term interferogram is mainly dominated by differential effects originating from changes in soil moisture and/or vegetation wet biomass (e.g. due to growth or senescence), as noticeable from the subtle phase differences between Fig.6 a)-b). These differential effects also affect the estimation of constant and linear phase offsets to a much larger extent in the case of the direct processing approach. Therefore, an appropriate comparison between the two processing strategies needs to rely on deterministic targets, such as buildings or ideally corner reflectors. Particularly, the identification of persistent bright scatterers has been carried out by analysing their amplitude scintillation within the stack of SAR images. Each scatterer has been isolated within a neighbourhood of 60 $\mathrm{m} \times 60 \mathrm{~m}$ to avoid excessive clustering of nearby scatterers. For each of the 167 identified persistent bright scatterers, the RMS error of the residual phase obtained with the direct and graph-based approach has been compared in Fig.7. The latter
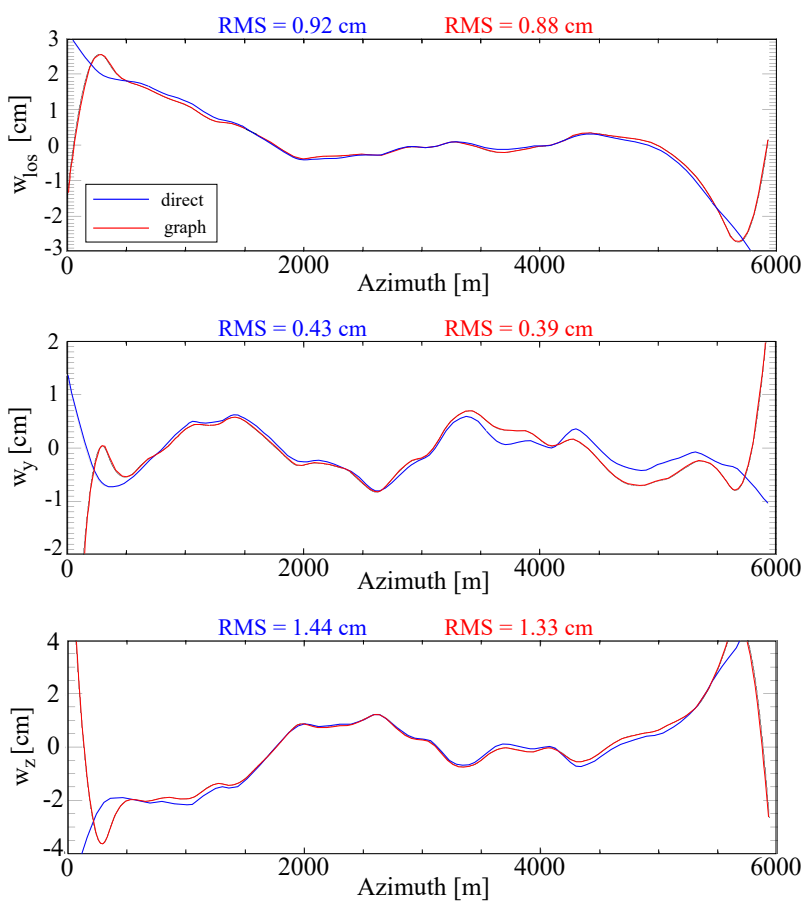

Fig. 5: Estimated RBEs projected along the line-of-sight $\left(\boldsymbol{w}_{\text {los }}\right)$, horizontal $\left(\boldsymbol{w}_{y}\right)$, and vertical $\left(\boldsymbol{w}_{z}\right)$ directions. Blue lines correspond to the RBEs estimated with MS (direct processing). Red lines represent the post-processing residual baseline correction obtained in the graph-based processing of $\left(s_{4,0}, s_{7,0}\right)$. RMS stands for Root Mean Square.

shows that the RMS error of the DEM flattened phase is considerably reduced by the proposed graph-based approach.

\section{CONCLusion}

A refined processing strategy including a post-processing motion compensation algorithm has been herein presented. The approach exploits the high accuracy of the residual baseline error estimates obtained by applying a generic residual motion compensation algorithm (e.g. multisquint) to interferometric pairs slightly affected by decorrelation. In case of heavily decorrelated scenarios, as the one presented in this paper, the proposed approach allows for obtaining more accurate residual baseline error estimates compared to the sole use of the multisquint algorithm as a residual motion correction strategy. The proposed strategy is particularly suited to the processing of collections of temporally separated tomographic acquisitions as long as the coherence magnitude is reasonably high over small spatial and/or temporal interferometric pairs. This provides and effective but simple way of obtaining accurate residual baseline error estimates for long spatial and/or temporal interferometric pairs. However, a drawback of the presented methodology is that the improvement of the interferometric phase quality is in turn dependent on the accuracy of the residual baseline errors obtained in the pairwise processing. The proposed post-processing residual baseline correction works on the top of data-driven residual 


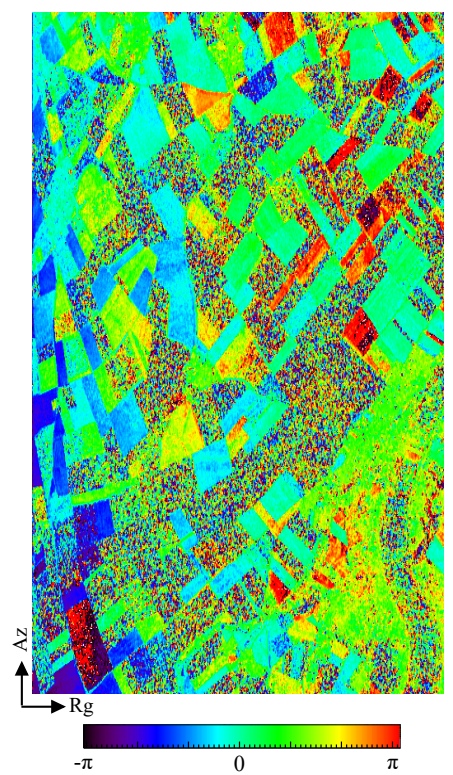

a)

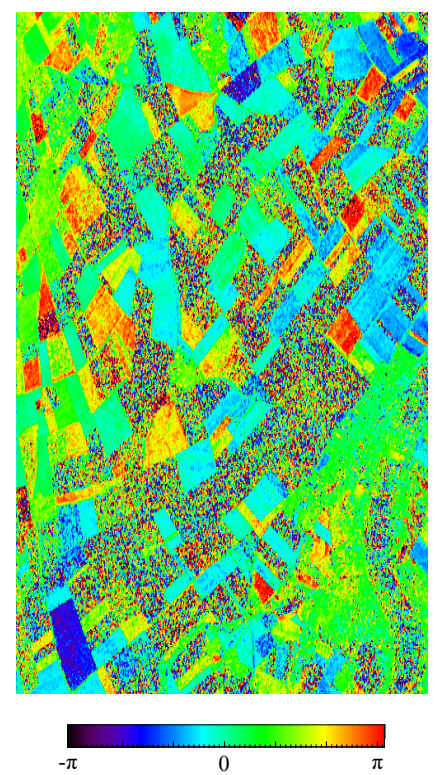

b)

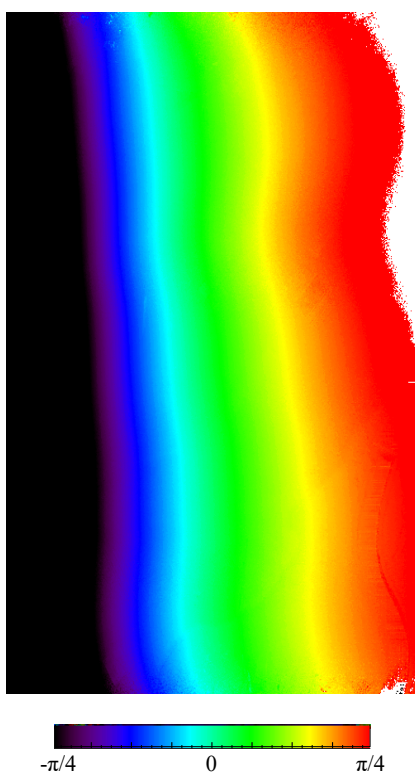

c)

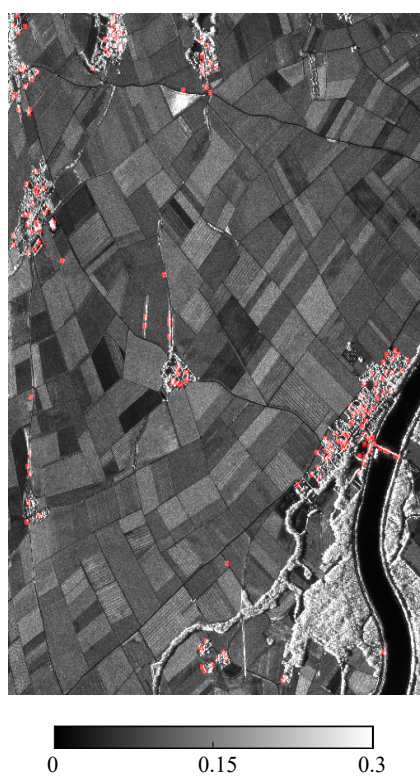

d)

Fig. 6: From left to right: HH interferometric phase between $\left(s_{4,0}, s_{7,0}\right)$ processed with the direct (Fig.a) and graph-based approach (Fig.b). Fig.c phase difference between the slave image $s_{7,0}$ processed with the direct and graph-based approach. Fig.d HH amplitude image of the test area. Red squares identify the stable scatterers used for the RMS analysis in Fig.7.

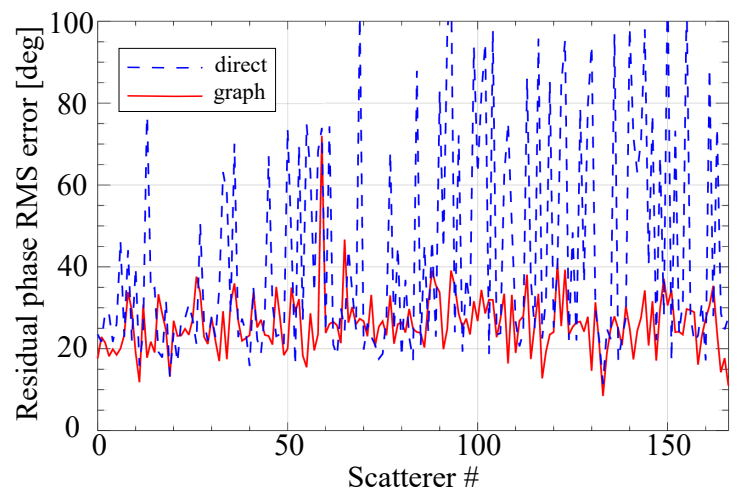

Fig. 7: Residual phase RMS error for the stable scatterers identified in Fig.6-d. Colour coding discriminate the direct (blue) and the graph-based (red) approach.

baseline correction algorithms and, therefore, it suffers from the same drawbacks when the observed scene is heavily decorrelated over small temporal and/or spatial baselines.

Future work might extend the applicability of the proposed strategy to SAR data sets acquired at C- and/or X-band, for which the impact of decorrelation is more pronounced. The main challenge is to accommodate differences between the pairwise processing geometry (e.g. the squint angle) and that of the entire SAR data stack. This would require to transform the pairwise RBE estimates into a global Cartesian coordinate system prior to their accumulation.

\section{REFERENCES}

[1] R. Bamler and P. Hartl, "Synthetic aperture radar interferometry," Inverse problems, vol. 14, no. 4, pp. 1-54,
January 1998.

[2] P. Prats, A. Reigber, J. J. Mallorquí, R. Scheiber, and A. Moreira, "Estimation of the temporal evolution of the deformation using airborne differential SAR interferometry," IEEE Transactions on Geoscience and Remote Sensing, vol. 46, no. 4, pp. 1065-1078, March 2008.

[3] A. Reigber and R. Scheiber, "Airborne differential SAR interferometry: First results at L-band," IEEE Transactions on Geoscience and Remote Sensing, vol. 41, no. 6, pp. 1516-1520, June 2003.

[4] A. Reigber, P. Prats, and J. J. Mallorqui, "Refined estimation of time-varying baseline errors in airborne SAR interferometry," IEEE Geoscience and Remote Sensing Letters, vol. 3, no. 1, pp. 145-149, January 2006.

[5] A. Reigber, R. Scheiber, M. Jager, P. Prats-Iraola, I. Hajnsek et al., "Very-high-resolution airborne synthetic aperture radar imaging: Signal processing and applications," Proceedings of the IEEE, vol. 101, no. 3, pp. 759-783, November 2013.

[6] K. A. C. De Macedo, R. Scheiber, and A. Moreira, "An autofocus approach for residual motion errors with application to airborne repeat-pass sar interferometry," IEEE Transactions on Geoscience and Remote Sensing, vol. 46, no. 10, pp. 3151-3162, October 2008.

[7] A. Ferretti, C. Prati, and F. Rocca, "Permanent scatterers in sar interferometry," IEEE Transactions on Geoscience and Remote Sensing, vol. 39, no. 1, pp. 8-20, January 2001.

[8] J. M. Harris, J. L. Hirst, and M. J. Mossinghoff, Combinatorics and graph theory. New York, Springer, 2008, vol. 2. 